\title{
Analysis of Halogen and Other $\sigma$-Hole Bonds in Crystals
}

\author{
Peter Politzer * and Jane S. Murray * \\ Department of Chemistry, The University of New Orleans, New Orleans, LA 70148, USA \\ * Correspondence: ppolitze@uno.edu (P.P.); jsmurray@uno.edu (J.S.M.)
}

Received: 10 January 2018; Accepted: 10 January 2018; Published: 17 January 2018

Schneider has observed that [1]: “ . . the chemistry of the last century was largely the chemistry of covalent bonding, whereas that of the present century is more likely to be the chemistry of noncovalent binding." Given the speed with which everything moves now, perhaps half-century is more appropriate. In any case, the prediction is so far borne out by the avalanche of papers that have appeared in recent years dealing with halogen bonding and its various analogues, including the venerable hydrogen bond.

The entertaining arguments about the detailed natures of noncovalent interactions will probably never end (just as they have not ended for covalent bonds). They stem from basically different points of view and approaches to the issues; furthermore, they possess the happy feature that in many instances it is not possible to prove anyone either right or wrong!

However, this need not and will not impede important advances in applying noncovalent interactions in areas ranging from pharmacology to molecular electronics. In this special issue, we are pleased to present contributions from some of the most active researchers in the field of halogen bonding and other analogous interactions.

Conflicts of Interest: The authors declare no conflict of interest.

\section{References}

1. Schneider, H.-J. Binding Mechanisms in Supramolecular Complexes. Angew. Chem. Int. Ed. 2009, 48, 3924-3977. 